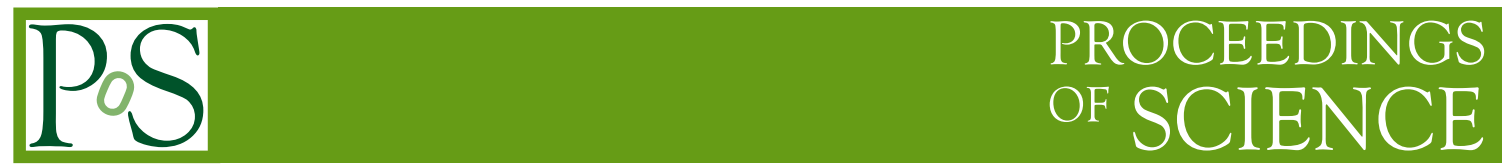

\title{
Exclusive and diffractive physics results from CMS
}

\section{Robert Ciesielski*}

On behalf of the CMS Collaboration

The Rockefeller University, 1230 York Avenue, New York, NY 10065, USA

E-mail: robert.ciesielski@rockefeller.edu

We report on the CMS measurements of exclusive and diffractive processes, including the latest results on the exclusive and diffractive production of dijets, dimuons, dielectrons and diphotons in pomeron- and photon-induced collisions in proton-proton interactions at $\sqrt{s}=7 \mathrm{TeV}$. The data are compared to various theoretical predictions.

36th International Conference on High Energy Physics,

July 4-11, 2012

Melbourne, Australia

${ }^{*}$ Speaker. 


\section{Introduction}

A significant fraction (approx. 20-30\%) of the total inelastic proton-proton cross section at high energies can be attributted to diffractive interactions, characterized by the presence of at least one large rapidity gap (LRG) in the final state. The LRG, defined as a region in pseudorapidity devoid of particles, is presumed to be formed by a color-singlet exchange carrying quantum numbers of the vacuum, often referred to as Pomeron $(\mathbb{P})$. Figure 1 shows the main types of diffractive processes: single dissociation (SD), double dissociation (DD) and central dissociation (CD).

Inclusive ( $\left(\mathrm{soft}^{1}\right)$ diffractive interactions have been traditionally described by models based on Regge theory. These phenomenological models suffer from large uncertainties when extrapolated to higher energies and need additional tuning at the LHC. On the other hand, diffractive processes involving a hard parton-parton scattering can be calculated within perturbative QCD (pQCD). Such processes are characterized by the presence of high- $p_{T}$ jets or high-mass particles in the final state in addition to the rapidity gap. The $\mathrm{PQCD}$ predictions, however, are sensitive to the soft rescattering between spectator partons, which significantly suppresses the formation of the rapidity gap (i.e., it suppresses diffractive cross sections). The LHC data allow to test a fundamental aspect of QCD, namely the interplay between soft and hard contributions to an interaction. Its good understanding at high energies is crucial for the proper modeling of the final state of Minimum-Bias events, and can help improve the simulation of e.g. the underlying event, pile-up events, and the measurement of the machine luminosity at the LHC.

Another class of processes with a LRG in the final state is exclusive $\gamma \gamma$ production of lepton pairs, $p p \rightarrow p p l^{+} l^{-}$, with $l$ being a muon or an electron. The cross section for this QED process is known with very good accuracy and the process has been proposed for a complementary calibration of the luminosity measurement in $p p$ collisions. It also provides an excellent control sample for exclusive QCD processes whose theoretical predictions are less certain, such as exclusive $\gamma \gamma$ production, $p p \rightarrow p p \gamma \gamma$, or, in the future, exclusive Higgs boson production, $p p \rightarrow p p H$.

This paper reviews recent results on diffractive and exclusive processes obtained by the CMS experiment at the LHC, based on the early data collected in 2009 and 2010 when the LHC was running in a low pile-up scenario, most suitable for event selection based on the LRG signature. Detectors used for the studies were the tracking devices and calorimeters in the central region $(|\eta|<2.4$ and $|\eta|<3$, respectively), Hadron-Forward calorimeters (HF) covering the region of $3<|\eta|<5$ on both sides of the interaction point, and the Beam Shower Counters (BSC) attached to the front of the HF detectors.

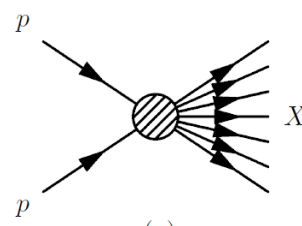

(a)

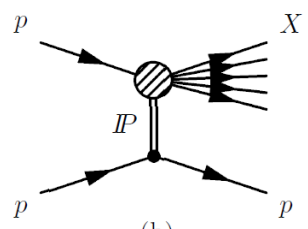

(b)

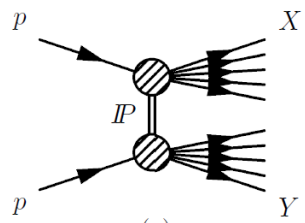

(c)

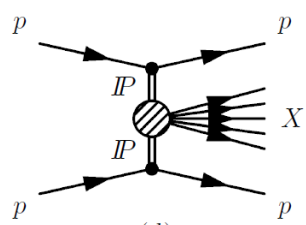

(d)

Figure 1: Schematic diagrams of (a) non-diffractive, $p p \rightarrow X$, and diffractive processes with (b) singledissiociation, $p p \rightarrow X p$ or $p p \rightarrow p Y$ (not shown), (c) double-dissociation, $p p \rightarrow X Y$, and (d) centraldissociation, $p p \rightarrow p X p$. The $X(Y)$ represents a dissociated-proton or a centrally-produced hadronic system.

\footnotetext{
${ }^{1}$ with no hard scale, which is needed for perturbative calculations.
} 


\section{Diffractive processes}

Among the very first results obtained by CMS was the evidence for observation of SD diffractive processes[1]. This result was based on data from dedicated runs with negligible pile-up events, at $\sqrt{s}=0.9,2.36$ and $7 \mathrm{TeV}$. Events were selected by requiring a signal in any of the BSC scintillators (Minimum Bias trigger) and the presence of the reconstructed event vertex. SD events appear as a peak around zero in the energy and multiplicity distributions of the HF calorimeter, reflecting the presence of the LRG in HF. Figure 2 shows these distributions for the $7 \mathrm{TeV}$ data. They are compared to Monte Carlo (MC) predictions of PYTHIA6, PYTHIA8 and PHOJET, with and without diffraction included. The agreement is poor and reflects a general feature of pre-LHC MC generators, which are not able to describe the new data at $7 \mathrm{TeV}$.
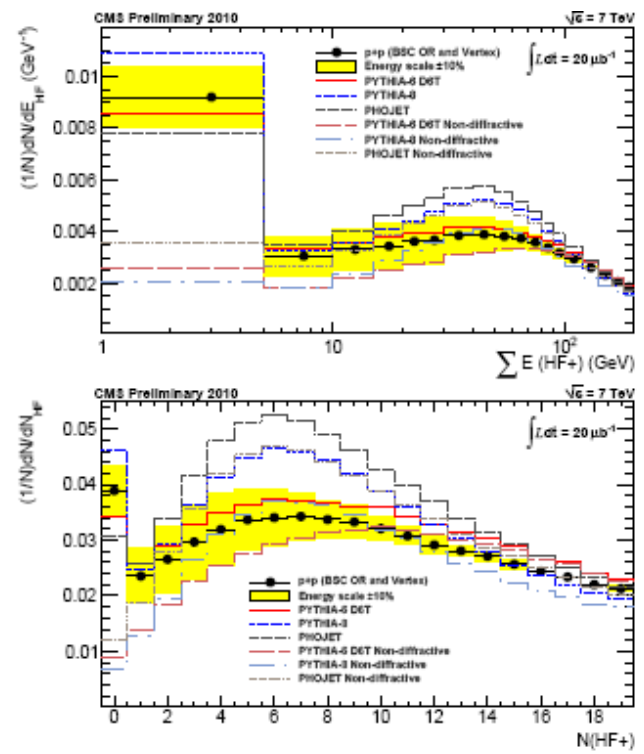

Figure 2: Uncorrected distributions of the energy sum (top) and the tower multiplicity (bottom) in one of the HF calorimeters at $\sqrt{s}=7 \mathrm{TeV}$.

Diffraction with a hard scale has been recently studied by selecting events dominated by a contribution from diffractive di-jet production[2]. The analysis is based on dedicated runs at $\sqrt{s}=7$ $\mathrm{TeV}$, corresponding to an integrated luminosity of $L=2.7 \mathrm{nb}^{-1}$ and negligible pile-up. The inclusive di-jet sample has been selected by requiring at least two jets with $p_{T}>20 \mathrm{GeV}$ and $|\eta|<4.4$, reconstructed using the anti- $k_{T}$ algorithm with a radius of $R=0.5$. Di-jet events have been studied as a function of the variable $\widetilde{\xi}$, calculated from all energy deposits in the detector (above threshold). Diffractive events populate the region of low values of $\widetilde{\xi}$, which for the SD process approximates the incoming-proton momentum fraction transferred to the diffractive exchange. The relation between $\tilde{\xi}$ and a LRG, defined by requiring no individual energy deposits above $4 \mathrm{GeV}$ in one of the two HF calorimeters, is illustrated in Fig. 3(left), showing the distribution of events as a function of $\tilde{\xi}$ before (full dots) and after (open dots) applying the LRG condition. The data are well
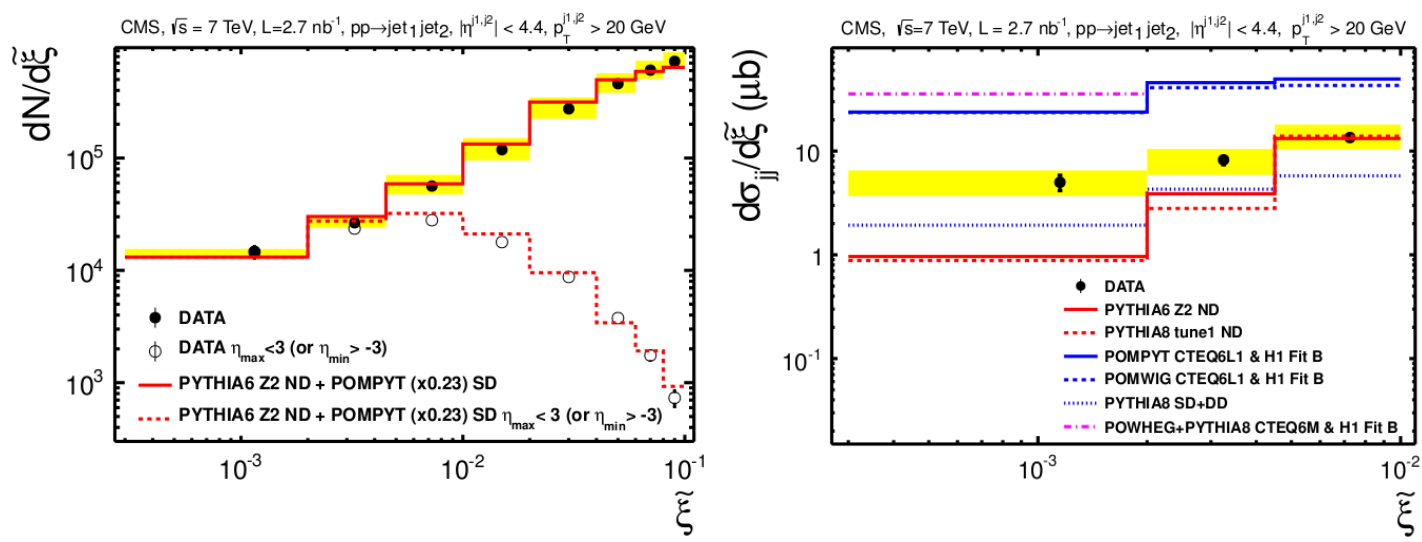

Figure 3: The distribution of events as a function of $\widetilde{\xi}$ obtained before and after the LRG requirement (left) and the differential cross section for dijet production as a function of $\widetilde{\xi}$ (right), compared to MC predictions. 
described by a combination of SD (POMPYT) and non-diffractive (PYTHIA6-Z2) MC samples, whose relative yields were obtained from a fit to the $\widetilde{\xi}$ distribution in the inclusive di-jet sample. Cross sections for inclusive di-jet production, corrected to the stable-particle level, are shown in Fig. 3(right), in three bins of $\widetilde{\xi}$, for $\widetilde{\xi}<0.01$. Results are compared to non-diffractive (PYTHIA6 and PYTHIA8) and diffractive (POMPYT SD, POMWIG SD and PYTHIA8 SD+DD) MC models, as well as to NLO calculations of the SD cross section from POMHEG. An excess of events in the low $-\widetilde{\xi}$ region w.r.t. non-diffractive samples is clearly seen. In the lowest $\widetilde{\xi}$ bin $(0.0003$ $<\widetilde{\xi}<0.002$ ) the diffractive PYTHIA 8 prediction is a factor of $\sim 2$ below the data. The LO predictions from POMPYT and POMWIG, and NLO predictions from POMHEG (all three based on dPDFs extracted from HERA experiments) overestimate the data by a factor of $\sim 5$ and $\sim 10$, respectively. After correcting for the DD contribution, these factors can be converted into an upper limit on the gap survival probability, which ranges from $\approx 0.08 \pm 0.04$ (NLO) to $\approx 0.12 \pm 0.05$ (LO), in agreement with results obtained at Tevatron energies.

CMS has also studied the hard diffractive production of $W$ and $Z$ bosons [3], using the full 2010 dataset $\left(L=36 \mathrm{pb}^{-1}\right)$. Inclusive samples of events with leptonic decays of $W$ and $Z$ bosons was selected with the following criteria[4]: isolated lepton with $p_{T}>25 \mathrm{GeV}$ and $|\eta|<1.4 ; E_{T}>30$ $\mathrm{GeV}$ and $M_{W, Z}>60 \mathrm{GeV}$. The analysis provided an extensive study of the forward energy flow and central charged-particle multiplicities in the inclusive sample ${ }^{2}$, leading to a conclusion that none of the MC simulations is able to simultaneously describe the central and forward rapidity region in collisions at $\sqrt{s}=7 \mathrm{TeV}$. In addition, the LRG events were selected by requiring no individual energy deposit above $4 \mathrm{GeV}$ in one of HF calorimeters. The fraction of LRG events extracted from the data amounts to $1.46 \pm 0.09$ (stat.) \pm 0.38 (syst.)\% and $1.60 \pm 0.25$ (stat.) \pm 0.42 (syst.)\%, for $W$ and $Z$ events, respectively. Figure 4(left) presents the distribution of the energy deposited in HF detectors, for $W$ events, compared to the predictions of non-diffractive PYTHIA6 and PYTHIA 8 MC simulations. LRG events populate the lowest bin, where the variation of MC predictions is large. The diffractive component in the LRG sample was estimated by studying hemisphere
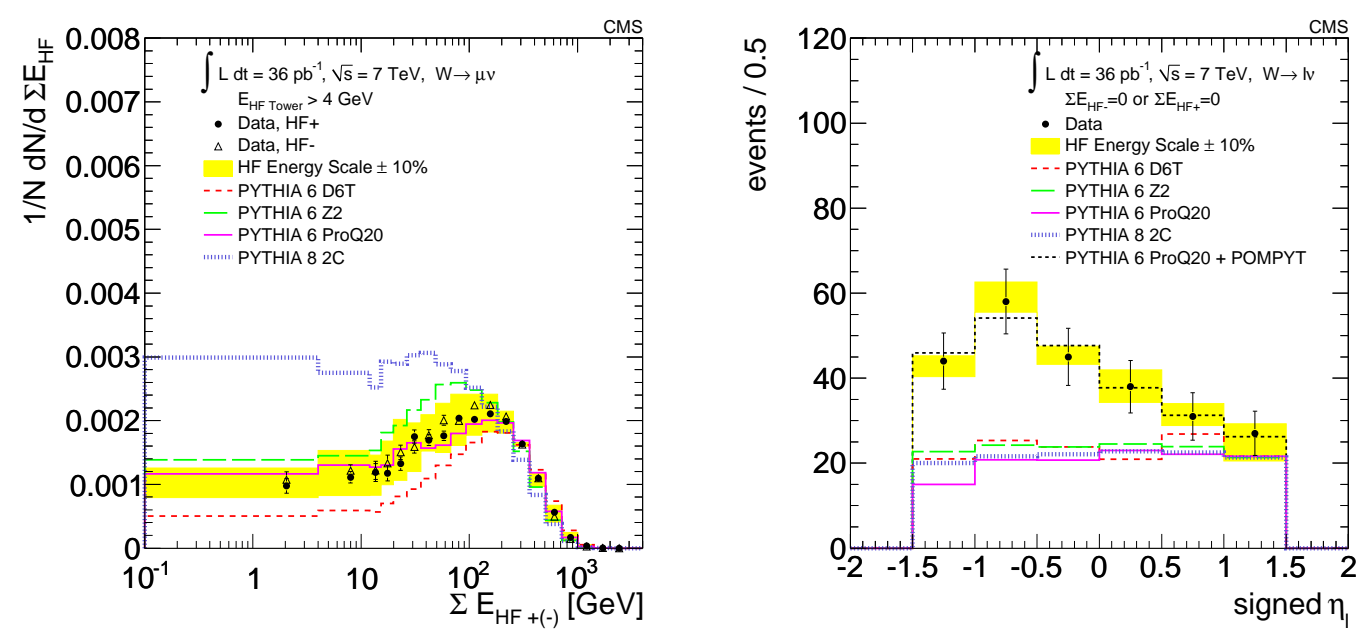

Figure 4: Uncorrected distributions of the energy sum in HF detectors (left) and the signed lepton pseudorapidity for events with a LRG signature (right), for $W \rightarrow \mu v$ production compared to MC predictions.

\footnotetext{
${ }^{2}$ a hard process with a colorless final state involving a $W$ or $Z$ boson provides a straightforward separation of the soft underlying event from the hard interaction, allowing to test the MC modelling of the non-perturbative component.
} 

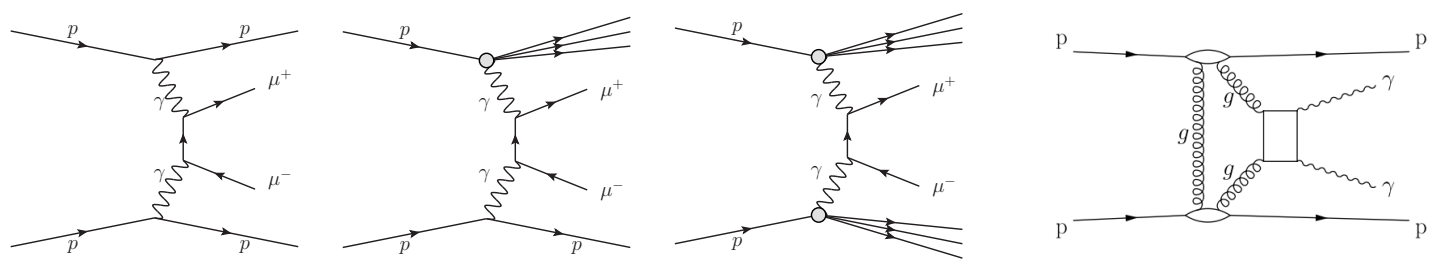

Figure 5: Schematic diagrams of the exclusive (left), single-dissociative (center left), double-dissociative (center right) di-photon production of muon pairs in $p p$ collisions, as well as of central exclusive $\gamma \gamma$ production, $p p \rightarrow p p \gamma \gamma$ (right).

correlations between the gap and the $W$ boson. Figure 4(right) shows the distribution of the signed charged-lepton pseudorapidity, $\eta_{l}$, defined to be positive (negative) if the gap and the lepton are in the same (opposite) hemisphere. The observed $\eta_{l}$ asymmetry is predicted by the POMPYT MC simulation of SD diffractive $W$ production. The fraction of diffractive events in the LRG samples was extracted from a fit with ND PYTHIA6 and SD POMPYT simulations and amounts to $f_{S D}=50 . \pm 9.3$ (stat.) \pm 5.2 (syst)\%. This result provides the first evidence of diffractive $W$ boson production at the LHC.

\section{Exclusive di-lepton production and search for exclusive di-photon production}

A measurement of exclusive di-photon production of muon pairs has been performed at $\sqrt{s}=7$ $\mathrm{TeV}$, using muon and tracking detectors and the full 2010 dataset of $L=40 \mathrm{pb}^{-1}$ [5]. Di-muon pairs with invariant mass of $M_{\mu \mu}>11.5 \mathrm{GeV}, p_{T}>4 \mathrm{GeV}$ and $|\eta|<2.1$, originating from the common event vertex were selected. To enhance exclusivity, no other activity within $2 \mathrm{~mm}$ from the vertex was allowed (track veto). The selected sample consists of events of exclusive $\mu \mu$ production (Fig. 5(left)), as well as semi-exclusive $\mu \mu$ production (Fig. 5(center)), in which the dissociated proton escapes detection in the central detector. Figure 6 shows the distributions of acoplanarity and the $p_{T}$ of the di-muon pair, compared to LPAIR MC predictions for exclusive and semi-exclusive production, normalized to the data based on a fit described below. Good agreement between data and the simulation is observed. The cross section for exclusive $\mu \mu$ production was extracted from a fit to the $p_{T}(\mu \mu)$ distribution, with free parameters describing the
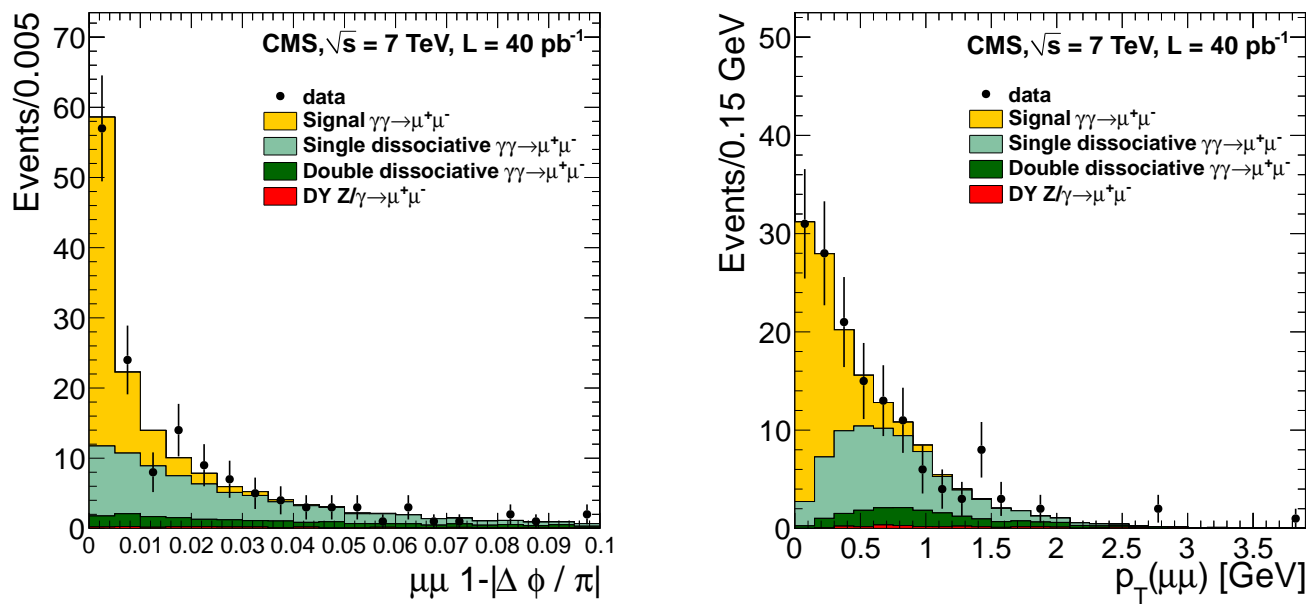

Figure 6: Distributions of the acoplanarity, $1-\Delta \phi / \pi$, (left) and the $p_{T}$ of the di-muon system, $p_{T}(\mu \mu)$, (right), compared to MC predictions with yields determined from a fit to the $p_{T}(\mu \mu)$ spectrum. 
signal and proton-dissociative yields relative to the LPAIR prediction, and the correction to the shape of the proton-dissociative sample (negligible). The cross section has been measured to be: $\sigma_{\text {excl. }}=3.38_{-0.55}^{+0.58}$ (stat) \pm 0.16 (syst.) \pm 0.14 (lumi. $) p b$, in good agreement with the LPAIR prediction within uncertainties.

The analysis of exclusive $\gamma \gamma$ production of electron pairs was performed simultaneously with a search for central exclusive $\gamma \gamma$ production (Fig. 5 (right)), using the full 2010 dataset $\left(L=36 \mathrm{pb}^{-1}\right)[6]$. Event selection for the di-electron sample required one electron and one positron with $p_{T}>5.5 \mathrm{GeV}$ and $|\eta|<2.5$, originating from a common event vertex, and no other particle detected in the region of $|\eta|<5.2$. The two electrons were required to be separated by more than $2.5 \mathrm{rad}$ in $\phi$. Due to limited statistics, driven by the exclusivity criterion $(\varepsilon \simeq 0.145 \pm 0.008)$, no separation between exclusive and semi-exclusive processes was performed. After all selections, 17 candidates for

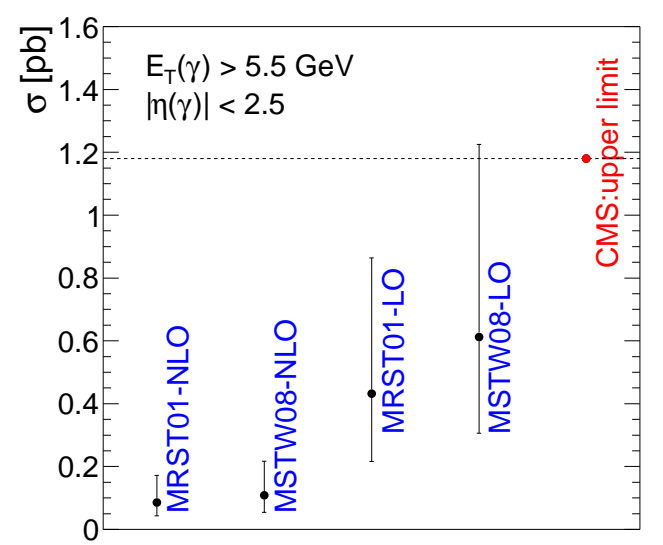

Figure 7: The $p p \rightarrow p p \gamma \gamma$ cross section limit compared to four different theoretical predictions. electron-pair production were observed in the data, in excellent agreement with the LPAIR MC predition of $16.5 \pm 1.7$ (theory) \pm (stat.) events. The result provides a validation of the experimental procedure for the exclusive $\gamma \gamma$ search.

No di-photon events have survived the selection described above, leading to an upper limit for exclusive and semi-exlusive $\gamma \gamma$ production of $\sigma_{\gamma \gamma}<1.3 \mathrm{pb}$ at 95\% CL. A comparison of the extracted limit to the predictions of the ExHume MC with several LO and NLO PDF sets is shown in Fig. 7. While the current result provides some constraints on LO predictions, more data are needed to test the NLO calculations.

\section{Summary}

The recent results on diffractive and exclusive processes studied with the central CMS detector, using the LRG signature, have been presented and compared to MC predictions.

\section{References}

[1] CMS Collaboration, Observation of diffraction in proton-proton collisions at 900 and $2360 \mathrm{GeV}$ centre-of-mass energies at the LHC, CMS-PAS-FWD-10-001, Observation of diffraction in proton-proton collisions at $7 \mathrm{TeV}$ centre-of-mass energies at the LHC, CMS-PAS-FWD-10-007.

[2] CMS Collaboration, Observation of a diffractive contribution to dijet production in proton-proton collisions at $\sqrt{s}=7 \mathrm{TeV}$, CMS-FWD-10-004, submitted to Phys. Rev. D [arXiv:1209.1805].

[3] CMS Collaboration, Eur. Phys. J. C72 (2012) 1839 [arXiv:1110.0181].

[4] CMS Collaboration, JHEP 01(2011) 080 [arXiv:1012.2466].

[5] CMS Collaboration, JHEP 01(2012)052 [arXiv:1111.5536].

[6] CMS Collaboration, Search for exclusive or semi-exclusive photon pair production and observation of exclusive and semi-exclusive electron pair production in pp collisions at $\sqrt{s}=7 \mathrm{TeV}$,

CMS-FWD-11-004, accepted by JHEP [arXiv:1209.1666]. 